\title{
INTEGRATING AHP INTO EUNETHTA CORE MODEL: THE DECISION- ORIENTED HEALTH TECHNOLOGY ASSESSMENT (DOHTA) METHOD
}

\section{ABSTRACT}

Health technology assessment (HTA) refers to the systematic evaluation of properties, effects, and/or impacts of health technology. It is a multidisciplinary process to evaluate the social, economic, organizational and ethical issues of a health intervention or health technology. The main purpose of conducting an assessment is to inform a policy decision making. Indeed, HTA is a multidimensional and multidisciplinary assessment process, aimed at supporting decisions pertaining to the allocation of resources.

The EUnetHTA Core Model ${ }^{\circledR}$ is a well-known and commonly used tool for structuring the evaluation of innovative health technologies. It is based on assessment elements that describe the technology or the consequences of its use by supplying the information needed to decide on the use or non-use of any selected technology

In order to empower decision makers to choose more knowingly between the different alternatives being considered, offering them a more precise and more structured output as well as contextualized evidence for a specific technology, we developed a standardized methodological approach that integrates Analytic Hierarchy Process (AHP) with the EuNetHTA Core Model ${ }^{\circledR}$, that we called Decision-Oriented HTA(doHTA).

Compared to the Core Model ${ }^{\circledR}$, doHTA supplies a more timely as well as contextualized evidence for a specific technology, making it possible to obtain data which are more relevant and easier to interpret, and therefore more useful for decision makers to make investment choices with greater awareness.

DoHTA has been devised mainly to address decision-making issues at hospital levels (i.e. pertaining the decision about implementing or not-implementing a specific, already marketed, health technology). However, its mathematical framework offers the possibility to be used in different settings ( $R \& D$, pricing and reimbursement, etc.) and in combination with other analytical methods (such as Markov models, MonteCarlo simulation, Deterministic Sensitivity Analysis).

Keywords: Health Technology Assessment, hospital decision-making, doHTA.

\section{Introduction}

The application of HTA methodology at, so called, meso-level and in particular its implementation at hospital level (defined as Hospital-Based Health Technology Assessment (HB-HTA)), is essential when considering the adoption or rejection of health technologies (HTs) in a hospital, as it is aimed at contextualizing both evidences and 
decisions. However, available scientific evidence cannot always supply useful answers to the questions raised by local decision makers.

\section{Hypotheses/Objectives}

In order to address issues raised by hospital's decision-makers, we devised the doHTA method to be used as a tool for guiding and supporting decision about the introduction of innovative health technologies.

\section{Research Design/Methodology}

The doHTA method is the combination of EUnetHTA core model and AHP.

Using the EUnetHTA Core Model ${ }^{\circledR}$ as guidance, it is possible to identify which specific "assessment element" may bring a piece of knowledge and thus translated into an indicator of the decisional structure. By means of scientific literature review, beyond acquiring a deeper knowledge of the question under examination and gathering scientific evidence about the alternative health technologies, their implementation and outcomes, we identify all the components of the decisional hierarchy structure and, in agreement with the AHP method, their topological arrangement within it.

Once a hierarchical structure has been made available, AHP method and tasks can be easily accomplished.

\section{Limitations}

Since the interviewees have different competences and roles (that is, belonging to different professional and expertise areas), there may be room for judgement's bias. A simplistic application of the geometric mean as per the computation of the global weights' system and alternatives' priorities can lead indeed to a biased evaluation, due to the possible imbalance of experts involved. A possible solution is represented by clustering each professional with respect to his/her professional area, computing both weights and priorities for each professional area and then computing the global ones. Moreover, it becomes essential to involve in the assessment all those professionals representing hospital sectors potentially affected by the implementation of the new technology.

\section{Conclusions}

We have tested the doHTA method on a variety of health technologies (from hospitalwide services to multipurpose tools to very specialized devices) to check its feasibility, adaptability and scalability. Our method allows a structured and more precise output, giving the decision makers the possibility to choose more knowingly between the different considered alternatives, often in a very short time (as they have been provided with a clear and instantaneous depiction of the whole evaluation, making their final choice easier and faster). Moreover, it can be used in different settings (R\&D, pricing and 
reimbursement, etc.) even because, thanks to its mathematical framework, it can be combined with other analytical methods (such as Markov models, MonteCarlo simulation, Deterministic Sensitivity Analysis).

\section{Key References}

- European Network for Health Technology Assessment (EUnetHTA). HTA core model for medical and surgical interventions. Available from: http://www.eunethta.eu/outputs/hta-core-model-medical-and-surgical-interventions10r [Accessed May 27, 2014].

- Saaty TL. Decision making with the analytic hierarchy process. Int. J. Services Sciences 2008;1;1;83-98.

- Battista RN, Hodge MJ. The evolving paradigm of health technology assessment: Reflections for the millennium. CMAJ 1999;18;160(10):1464-1467.

- Gagnon MP. Hospital-based health technology assessment: developments to date. Pharmacoeconomics. 2014 Sep;32(9):819-824. 